\title{
Post Traumatic Stress Disorder (PTSD) as an Over Activation of Sympathetic Nervous System: An Alternative View
}

\section{Eugene Lipov*}

Advanced Pain Centers S.C., 2260 W. Higgins Rd., Ste. 101, Hoffman Estates, IL, USA

\begin{abstract}
Stress and trauma are a part of the human condition. Trauma that is a catastrophic stressor, being defined as "outside the range of usual human experience" such as war, torture, rape, or natural disaster commonly leads to PTSD. Stressors can be physical, emotional, or a combination of both. Current treatment modalities for treating PTSD include conventional pharmaceuticals together with psychological therapeutics including cognitive processing therapy (CPT), exposure therapy and a number of other approaches. The success rates of combined therapies noted above is dangerously low, considering the acute need for successful treatments. The necessity to change the treatment paradigm from the conversional to more effective approaches cannot be over stated. Fortunately, sympathetic nervous system (SNS) modulation treatments are currently available, and are showing significant promise in the treatment of PTSD. One recent innovation is stellate ganglion block which is a selective sympathetic injection that can lead to normalization of over active SNS. In the near future, a combination of SNS based therapeutics with current approaches may lead to a marked improvement in the PTSD therapy success rates.
\end{abstract}

Keywords: Stress and trauma; Catastrophic stressor; Cognitive processing therapy; Social phobias

\section{Introduction}

Stress and trauma are a part of the human condition. Trauma that is a catastrophic stressor, being defined as "outside the range of usual human experience" such as war, torture, rape, or natural disaster, commonly leads to PTSD. Stressors can be physical or emotional, or a combination of both. Experiencing severe trauma is known to precipitate post-traumatic stress disorder. Current approaches for treating PTSD include conventional pharmaceuticals together with psychological therapeutics including cognitive processing therapy (CPT), exposure therapy and a number of other psychologically based approaches. Pharmaceutical approach to PTSD has entailed the use of selective serotonin reuptake inhibitors (SSRIs) and serotoninnorepinephrine reuptake inhibitors (SNRIs), those two classes of compounds have been the only ones to have A-level evidence (2 SSRIs are approved for this indication (Dep. of V.A. 2010).

The success of treating PTSD by utilizing conventional pharmaceuticals and psychological therapeutics including CPT and other, has been described by Dr. Hoge as being under 30\% in the military populations Hoge [1]. In above quoted study, Dr. Hoge reported a very low compliance rate with conventional treatments as a large part of difficulty treating PTSD patients. The success rate of under $30 \%$ is dangerously low considering the acute need for successful treatments. Perhaps the most painful demonstration of urgency for successful PTSD treatments is the spiraling suicide rate. Dr. Kemp assessed suicide rates in United States veterans in the year 2010, by doing a retrospective review. Her findings were grim, approximately 22 veterans have taken their life in 2010, as per Dr. Kemp's Suicide Data Report, 2012, Department of Veterans Affairs Mental Health Services Suicide Prevention Program [2]. In addition, this data is from 2010 and the suicide rate is continuing to increase. The US army has been actively trying to prevent suicides. In the course of US army search to prevent suicide escalation, it is becoming apparent that the suicide causes are due to multitude of interacting factors-such as job and personal stress, psychiatric conditions, and brain injuries-are contributing to a continuing epidemic of suicide among returning soldiers.

The necessity to change the treatment approach from the conventional to other more effective approaches cannot be over stated. Fortunately, more potent approaches to treat PTSD are available and may significantly improve success rates. Those approaches focus on the modulation of the sympathetic nervous system (SNS) and in combination with current therapies may lead to a significant improvement in PTSD response rates. The focus of this paper is normalization of SNS that is known to be chronically activated over the normal baseline levels in PTSD [3,4].

\section{Background of Sympathetic Nervous System as related to PTSD}

The sympathetic nervous system (SNS) is part of the autonomic nervous system. Its role is to mobilize body's resources under stress, to induce the fight-or-flight response. It is also constantly active at a basal level in order to maintain homeostasis. In large part, the activation of the SNS is accomplished by the increase of catecholamines, mainly epinephrine and norepinephrine. The role of norepinephrine in the brain is that of a neurotransmitter leading to arousal, selective attention, and vigilance which has been demonstrated in preclinical studies [5]. Specifically, elevated urinary norepinephrine has been identified among patients with PTSD [6]. Similarly, norepinephrine concentrations in cerebrospinal fluid (CSF) are significantly higher in subjects with PTSD than among healthy controls and have been correlated with the severity of PTSD symptoms [7]. Such notable increases in noradrenergic activity among subjects with PTSD suggest that reducing CNS noradrenergic activity could be effective, especially for arousal symptoms such as nightmares and startle reactions [8].

*Corresponding author: Eugene Lipov, Advanced Pain Centers S.C., 2260 W. Higgins Rd., Ste. 101, Hoffman Estates, IL USA, E-mail: elipovmd@aol.com

Received November 20, 2013; Accepted December 24, 2013; Published December 26, 2013

Citation: Lipov E (2013) Post Traumatic Stress Disorder (PTSD) as an Over Activation of Sympathetic Nervous System: An Alternative View. J Trauma Treat 3 : 181. doi:10.4172/2167-1222.1000181

Copyright: (c) 2013 Lipov E. This is an open-access article distributed under the terms of the Creative Commons Attribution License, which permits unrestricted use, distribution, and reproduction in any medium, provided the original author and source are credited. 


\section{Orally Active SNS Modulating Agents and their Effects on PTSD}

Orally active noradrenergic blocking or deactivating agents, that have been used to moderate an over-active SNS and reduce blood pressure include clonidine and prazosin. Both have been previously reported to have psychiatric effects on PTSD patients. This is welcome news, since studies of psychiatric medication used to augment SSRI treatment have generally been disappointing, with the exception of Prazosin, an alpha-1-adrenergic receptor antagonist, that has shown benefit in improving sleep through reduction of physiological reactivity associated with nightmares [9]. A double-blind placebo-controlled trials of Prazosin, demonstrated a dramatic $70 \%-80 \%$ reduction in combat-related PTSD nightmares) [10]. Prazosin also has been shown to reduce PTSD-related anxiety during the day comparable to that observed with the SSRIs [11].

The other SNS modulating agent, clonidine has been successfully used to treat hyperarousal symptoms of PTSD [12]. Previous psychiatric use of clonidine has been in the treatment of startle response associated with acute opioid withdrawal where adrenergic and anxiety component may be involved [13]. The similarity between the opioid withdrawal and startle response in PTSD was observed more than two decades ago. It was suggested by Dr. Kolb that clonidine might be useful in treating PTSD [14].

In addition to the orally active agents, sympathetic system can be modulated in a selective fashion by influencing peripheral sympathetic ganglions. This approach has been shown to significantly reduce PTSD symptoms. Below are the examples of those approaches.

\section{Modulation of Cervical Sympathetic Ganglions by Minimally Invasive Approaches and their Effects on PTSD}

\section{Endoscopic sympathetic block (ESB)}

Endoscopic sympathetic block was first reported in Finland by Dr. Telaranta to successfully treat severe anxiety and social phobias. Dr. Telaranta showed that clipping the sympathetic ganglia, via an endoscopic sympathetic block (ESB) at the second thoracic vertebra (T2), could produce psychiatric effects [15-17]. It was also noted that social phobias and PTSD share many common features-especially symptoms associated with over activity of the sympathetic nervous system (SNS), such as heart racing, hyper vigilance, and avoidance of painful psychic situations [16]. This was a significant advance; however ESB remains an invasive procedure. Fortunately, a percutaneous procedure became available to treat PTSD. This occurred following a review of PTSD related literature, where the author realized similarity of the stellate ganglion block (SGB) and ESB due to T-2 sympathetic nerve fibers passing thorough stellate ganglion and was able to predict the effect of SGB on PTSD [18].

\section{Stellate ganglion block ( SGB)}

The stellate ganglion block (SGB) is an anesthetic injection next to a group of nerves in the neck, called the stellate ganglion. The procedure has been used to treat chronic pain since 1925 and recent pilot studies have demonstrated great promise as a successful intervention for PTSD, among other indications. The first documented use of SGB for psychiatric effect was its use in the resolution of depression by bilateral SGB as noted by clinicians at the Cleveland Clinic in 1947 [19]. The first case study of successful use of the SGB to treat PTSD was reported by the author in 2008 [20]. Since 2008 a number of other institutions have utilized SGB for treatment of PTSD. Clinical results have been very encouraging. The institutions were: Walter Reed National Medical Center (Mulvaney 2010), Naval Medical Center San Diego [21], Tripler Army Medical Center [22], and Advanced Pain Centers $[20,23,24]$. Preliminary effectiveness rate seems to be 60 to $75 \%$ [24]. Other publications had limited number of patients thus success rates are difficult to determine, however a large study has recently been submitted for publication which demonstrated similar success rate to Lipov 2012 report (specifics cannot be referenced due to military protocols). Dr. Mulvaney study had two patients described in his 2010 report with a $100 \%$ success and Dr. Alino had four patients with $100 \%$ success. The above clinicians noted significant reductions of hypervigilance, insomnia, anxiety and the like.

Utilization of the sympathetic blockade has distinct advantages over other modes of PTSD treatments. Those advantages are: significant clinical effectiveness that is rapid and patient compliance is close to $100 \%$ since it does not depend on the patient returning for multiple treatments or needing to take medications at home. Finally, SGB seems to have significant effect on a suicidal patients as noted by $[20,22]$.

\section{Discussion}

Multiple different approaches, covering the entire spectrum of medicine, have been tried as a PTSD treatment. Interventions to help PTSD patients have spanned hyperbaric chamber, distal healing, theater of war (reading Greek tragedies to the patients), conventional psychotherapy, pharmacological therapy as well as the use of Ecstasy. Most results have been disappointing. Various hypothesis have been put forth to explain the possible etiology of PTSD and the proposed mechanisms of treatments. It seems that current trends are to have a biological explanation for PTSD etiology and its associated treatments. Modulation of the sympathetic system is that type of approach, since it fits the biological model and depends on well understood neurobiology and neuroanatomy. SGB is an approach that has been used by anesthesiologists for years to reduce the SNS activity, the very system known to be overactive in PTSD. Previous sympathetic blockade uses have been for reduction of SNS over activity in an extremity, for PTSD the effect is much more global. A hypothesis explaining neurobiological changes associated with PTSD onset and the effect of SGB leading to reversal of those changes is presented below.

The theory rests on a physiologic fact that humans respond to acute stress [25] and chronic stress [26] by secreting nerve growth factor (NGF) a representative of a neurotrophin family. NGF is involved in a variety of signaling events such as cell differentiation and survival, growth cessation, and apoptosis of neurons [27] Retrograde transport of NGF has been demonstrated from the intracerebral site to the stellate ganglion [28] Once in the stellate ganglion, NGF is known to promote sprouting (new nerve growth) at the sympathetic end terminals [29]. Sympathetic neurite outgrowth is known to secrete NE as a representative of SNS (Champlain 1972) [30]. Brain infusion of NGF in rats leads to perivascular NE increase [31]. PTSD patients have known increase in norepinephrine (NE) concentrations as discussed in the back ground section above. The final part of this hypothesis is the evidence that the application of local anesthetic to a sympathetic ganglion leads to the reduction of NGF [32] The NGF decrease leads to dying of new nerve outgrowth or sprouts [33], thus reversing the cascade and reducing NE levels. Reduction of NE following SGB has been shown in a human model by [34]. Thus the cascade is reversed, NE levels are reduced leading to reduction or elimination of PTSD symptoms [35-37]. 


\section{Conclusion}

A well recognized fact of sympathetic nervous system over activation as well as successful reduction of this very over activity may play a significant part in solving the scourge of Post Traumatic Stress Disorder (PTSD) plaguing United States of America, and every part of the planet. Utilization of orally active sympatholytic substances to reduce symptoms of PTSD is a modern day reality. Yet, orally active medications seem to have significant limitations in their effectiveness and use. Recent reports, from a number of independent teams, have documented significant improvements in PTSD symptoms following direct Sympathetic Nervous System (SNS) modulation by utilizing stellate ganglion blockade (SGB). The need to use different approaches to treat PTSD is clear, which approach or combination of approaches is not as clear. The emerging utility of SGB with possible combination of other modalities may, in the near future significantly impact the current epidemic of PTSD and PTSD related suicides. Early results following utilization of SGB for PTSD, including suicidal patients should lead to more formal studies to determine best patient selection and neuro biology of the effect.

\section{References}

1. Hoge CW (2011) Interventions for war-related posttraumatic stress disorder: meeting veterans where they are. JAMA 306: 549-551.

2. Kemp J, Bossarte R, Suicide Data Report, 2012,Department of Veterans Affairs Mental Health Services Suicide Prevention Program.

3. Lemieux AM, Coe CL (1995) Abuse-related posttraumatic stress disorder: evidence for chronic neuroendocrine activation in women. Psychosom Med 57: 105-115.

4. Krystal JH, Kosten TR, Southwick S, Mason JW, Perry BD, et al. (1989) Neurobiological aspects of PTSD: review of clinical and preclinical studies. Behavior Therapy 20: 177-198, Spring.

5. Southwick SM, Bremner JD, Rasmusson A, Morgan CA 3rd, Arnsten A, et al. (1999) Role of norepinephrine in the pathophysiology and treatment of posttraumatic stress disorder. Biol Psychiatry 46: 1192-1204.

6. Mason JW, Giller EL, Kosten TR, Harkness L (1988) Elevation of urinary norepinephrine/cortisol ratio in posttraumatic stress disorder. J Nerv Ment Dis 176: 498-502.

7. Geracioti TD Jr, Baker DG, Ekhator NN, West SA, Hill KK, et al. (2001) CSF norepinephrine concentrations in posttraumatic stress disorder. Am J Psychiatry 158: 1227-1230.

8. Taylor FB, Lowe K, Thompson C, McFall MM, Peskind ER, et al. (2006) Daytime prazosin reduces psychological distress to trauma specific cues in civilian trauma posttraumatic stress disorder. Biol Psychiatry 59: 577-581.

9. Department of V.A. Department of Veterans Affairs and Department of Defense. VA/DoD clinical practice guideline for management of post-traumatic stress.

10. Raskind MA, Peskind ER, Hoff DJ, Hart KL, Holmes HA, et al. (2007) A paralle group placebo controlled study of prazosin for trauma nightmares and sleep disturbance in combat veterans with post-traumatic stress disorder. Biol Psychiatry 61: 928-934.

11. Brady K, Pearlstein T, Asnis GM, Baker D, Rothbaum B, et al. (2000) Efficacy and safety of sertraline treatment of posttraumatic stress disorder: a randomized controlled trial. JAMA 283: 1837-1844.

12. Sutherland SM, Davidson JR (1994) Pharmacotherapy for post-traumatic stress disorder. Psychiatr Clin North Am 17: 409-423.

13. Harris AC, Gewirtz JC (2004) Elevated startle during withdrawal from acute morphine: a model of opiate withdrawal and anxiety. Psychopharmacology (Berl) 171: 140-147.

14. Kolb LC, Burris BC, Griffiths S (1984) Propranolol and clonidine in the treatment of posttraumatic stress disorders of war. In: van der Kolk BA (Ed.), PostTraumatic Stress Disorder: Psychological and Biological Sequelae. American Psychiatric Press, Washington, DC 97: 108.

15. Pohjavaara P, Telaranta T, Väisänen E (2003) The role of the sympathetic nervous system in anxiety: Is it possible to relieve anxiety with endoscopic sympathetic block? Nord J Psychiatry 57: 55-60.

16. Telaranta $T$ (2003) Psychoneurological applications of endoscopic sympathetic blocks (ESB). Clin Auton Res 13: I20-21.

17. Telaranta $T$ (1998) Treatment of social phobia by endoscopic thoracic sympathicotomy. Eur J Surg 27-32.

18. Lipov EG, Navaie M, Stedje-Larsen ET, Burkhardt K, Smith JC, et al. (2012) A novel application of stellate ganglion block: preliminary observations for the treatment of post-traumatic stress disorder. Mil Med 177: 125-127.

19. karnosh LJ, Gardner WJ (1947) The effects of bilateral stellate ganglion block on mental depression; report of 3 cases. Cleve Clin Q 14: 133-138.

20. Lipov EG, Joshi JR, Lipov S, Sanders SE, Siroko MK (2008) Cervical sympathetic blockade in a patient with post-traumatic stress disorder: a case report. Ann Clin Psychiatry 20: 227-228

21. Hickey AH, Navaie M, Stedje-Larsen ET, Lipov EG, McLay RN (2013) Stellate Ganglion Block for the Treatment of Posttraumatic Stress Disorder. Psychiatric Annals 43: 87-92.

22. Alino J, Kosatka D, McLean B, Hirsch K (2013) Efficacy of stellate ganglion block in the treatment of anxiety symptoms from combat-related post-traumatic stress disorder: a case series. Mil Med 178: e473-476.

23. Lipov EG, Navaie M, Stedje-Larsen ET, Burkhardt K, Smith JC, et al. (2012) A novel application of stellate ganglion block: preliminary observations for the treatment of post-traumatic stress disorder. Mil Med 177: 125-127.

24. Lipov E, Kelzenberg B (2012) Sympathetic system modulation to treat post-traumatic stress disorder (PTSD): a review of clinical evidence and neurobiology. J Affect Disord 142: 1-5.

25. Alleva E, Petruzzi S, Cirulli F, Aloe L (1996) NGF regulatory role in stress and coping of rodents and humans. Pharmacol Biochem Behav 54: 65-72.

26. Smith MA (1996) Hippocampal vulnerability to stress and aging: possible role of neurotrophic factors. Behav Brain Res 78: 25-36.

27. Snider WD (1994) Functions of the neurotrophins during nervous system development: what the knockouts are teaching us. Cell 77: 627-638.

28. Johnson EM Jr, Taniuchi M, Clark HB, Springer JE, Koh S, et al. (1987) Demonstration of the retrograde transport of nerve growth factor receptor in the peripheral and central nervous system. J Neurosci 7: 923-929.

29. Chen PS, Chen LS, Cao JM, Sharifi B, Karagueuzian HS, et al. (2001) Sympathetic nerve sprouting, electrical remodeling and the mechanisms of sudden cardiac death. Cardiovasc Res 50: 409-416.

30. De Champlain J, Van Ameringen MR (1972) Regulation of blood pressure by sympathetic nerve fibers and adrenal medulla in normotensive and hypertensive rats. Circ Res 31: 617-628.

31. Isaacson LG, Billieu SC (1996) Increased perivascular norepinephrine following intracerebroventricular infusion of NGF into adult rats. Exp Neurol 139: 54-60.

32. Takatori M, Kuroda Y, Hirose M (2006) Local anesthetics suppress nerve growth factor-mediated neurite outgrowth by inhibition of tyrosine kinase activity of TrkA. Anesth Analg 102: 462-467.

33. Gatzinsky KP, Thrasivoulou C, Campioni-Noack M, Underwood C, Cowen T (2004) The role of NGF uptake in selective vulnerability to cell death in ageing sympathetic neurons. Eur J Neurosci 20: 2848-2856.

34. Yokoyama M, Nakatsuka H, Itano Y, Hirakawa M (2000) Stellate ganglion block modifies the distribution of lymphocyte subsets and natural-killer cell activity. Anesthesiology 92: 109-115.

35. Gowing L, Farrell M, Ali R, White JM (2003) Alpha2 adrenergic agonists for the management of opioid withdrawal. Cochrane Database Syst Rev CD002024.

36. Kienbaum P, Peters J, Scherbaum N (2003) Convincing effects of clonidine on neurohumoral withdrawal symptoms during antagonist-supported detoxification of opioid addicts. Anesth Analg 97: 1547-1548.

37. Mulvaney SW, McLean B, de Leeuw J (2010) The use of stellate ganglion block in the treatment of panic/anxiety symptoms with combat-related post-traumatic stress disorder; preliminary results of long-term follow-up: a case series. Pain Pract 10: 359-365 